\title{
Generalized hyperbolic Ernst equations for an Einstein-Maxwell-Weyl field
}

\author{
Anastasios Tongas and Frank Nijhoff \\ Department of Applied Mathematics, University of Leeds, Leeds LS2 9JT, U.K.
}

November 5, 2018

\begin{abstract}
A new family of nonlinear partial differential equations is presented. They represent a generalization of the hyperbolic Ernst equations for an Einstein- Mawxell-Weyl field in general relativity. A Bäcklund transformation for the system of equations under consideration is given, and their direct relation to the complete Boussinesq hierarchy of soliton equations is illustrated.
\end{abstract}

\section{Introduction}

Plane symmetric spacetimes are of fundamental importance in Einstein's theory of gravitation. They describe the nonlinear interaction following the collision of plane fronted gravitational waves solely, or coupled with other fields. In this context the basis of the vacuum Einstein equations is formed by the hyperbolic version of the famous Ernst equation 11, 2. Many exact solutions of the Ernst equation and of its various extensions, which form the basis of the Einstein equations in the presence of certain fields, have been found 3. These solutions allow one to study the collisional interactions, indirectly. Assuming that the approaching waves are known, the direct problem is formulated by the characteristic initial value problem of the hyperbolic Ernst equations. For recent progress on the analysis of the preceding problem, as well as on the development of an effective integral representation of the solution in the interaction region, we refer to [4, 5, 6, 7. The underlying structure which has lent to the Ernst equation a prominent position as a nonlinear partial differential equation is the large number of the internal symmetries, as was originally suggested by Geroch [8, 9]. More recently in [10] it was shown that the infinitesimal Geroch transformations for stationary axisymmetric spacetimes extend to a large class of equations which reside in the $(2+1)$-dimensional Loewner system. The latter system encodes a variety of equations which from a modern perspective are considered to be integrable.

One of the outstanding universal features of integrable nonlinear evolution equations is the fact that they are not isolated objects, but they arise always with a structured infinite dimensional hierarchy of mutually compatible evolution equations. The latter has been exploited in many ways, leading to the intimate connection between the associated hierarchy of an integrable evolution equation and the representation theory of affine Lie algebras (and loop groups), see e.g. [11] and references therein. The point of view that one single equation in the hierarchy possesses a dominant character is misguided, and in fact, one should consider the infinite hierarchy of all equations together as being the integrable system.

It is remarkable that there exist circumstances in which the entire hierarchy of an integrable evolution equation can be encoded in a single equation. A prime example of such an equation was 
given in [12], and it is represented by a scalar fourth order partial differential equation (PDE). The entire hierarchy of the Korteweg-de Vries $(\mathrm{KdV})$ equations follows by systematic expansions from this scalar PDE. Moreover, in [13] it was shown that a proper generalization of this PDE incorporates the hyperbolic Ernst equation for a Weyl neutrino field in general relativity.

In this paper, a novel system of PDEs of fourth order in the independent variables is presented, which incorporates the hyperbolic Ernst equations for a source-free Maxwell field and a Weyl neutrino field. On the other hand this system represents in fact a consistent parameter-family of PDEs in the same spirit reported in [12, which encodes the entire hierarchy of Boussinesq soliton equations. To be more precise, the system of PDEs arises as the Euler-Lagrange equations for the variational problem associated with the Lagrangian density

$$
L=(u-v) \frac{\mathcal{F} \mathcal{E}}{\mathcal{J}^{2}}+m(v) \frac{\mathcal{F}}{\mathcal{J}}+n(u) \frac{\mathcal{E}}{\mathcal{J}} .
$$

The scalars quantities $\mathcal{F}, \mathcal{E}$ and $\mathcal{J}$ are given by

$$
\mathcal{F}=\boldsymbol{f}_{, u v} \wedge \boldsymbol{f}_{, u}, \quad \mathcal{E}=\boldsymbol{f}_{, u v} \wedge \boldsymbol{f}_{, v}, \quad \mathcal{J}=\boldsymbol{f}_{, u} \wedge \boldsymbol{f}_{, v},
$$

where $\boldsymbol{f}=\left(f_{1}, f_{2}\right)$. The components of $\boldsymbol{f}$, are complex functions of $(u, v) \in \mathbb{C}^{2}$, and $n$ and $m$ are complex parameter functions of the indicated arguments, unless otherwise stated. Partial differentiation is denoted by a comma or $\partial$, followed by the variable(s) with respect to which the differentiation has been performed. The Lagrangian $L$ remains invariant, molulo $L$ and null Lagrangians, under the projective group of linear fractional transformations on $\boldsymbol{f}$, i.e.

$$
f_{i} \longmapsto \frac{\alpha_{i 1} f_{1}+\alpha_{i 2} f_{2}+\alpha_{i 3}}{\alpha_{31} f_{1}+\alpha_{32} f_{2}+\alpha_{33}},
$$

$i=1,2$, and $\left(\alpha_{i j}\right) \in \mathrm{SL}(3, \mathbb{C})$. If in addition the parameter functions $n$ and $m$ are constants, then $L$ remains invariant under the following affine transformations:

$$
(u, v) \mapsto\left(\epsilon_{1} u+\epsilon_{2}, \epsilon_{1} v+\epsilon_{2}\right), \quad \epsilon_{1}, \epsilon_{2} \in \mathbb{C}, \epsilon_{1} \neq 0 .
$$

The above symmetries are called divergence symmetries of the associated variational problem, and every symmetry of this type is inherited as a Lie-point symmetry to the Euler-Lagrange equations, cf 14 .

The integrability of the Euler-Lagrange equations associated with $L$, will be demonstrated by the existence of the usual paraphernalia of the integrable models, such as of an associated linear representation (Lax pair) and a Bäcklund transformation, in sections 2 and 4 , respectively. The connection with the complete hierarchy of the Boussinesq soliton equations is addressed in section 3 , by exploiting the infinite symmetries of the field equations associated with the Lagrangian $L$. Section 5 deals with the reduction of the field equations to the Ernst equations of general relativity and the paper concludes in section 6 with discussion.

The continuous field equations and the derivation of the associated Lax pair and Bäcklund transformation originate in a compatible parameter family, of multi-field partial difference equations (P $\Delta \mathrm{Es}$ ); the two-dimensional discrete Boussinesq system. For the relevant constructions we use an intrinsic property which enjoys the discrete Boussinesq system and consists of its multi-dimensional consistency. Recently in [15], this property has been successfully exploited, to obtain a classification of one-field equations living on elementary quadrilateral faces. It can be argued that discrete integrable systems are equally important just as their continuous analogues, and in many respects 
their study has led to new insights into the structures behind the more familiar continuous systems. The notion of Bäcklund transformations and their associated Bianchi permutability theorems, which emerged in the transformation theory of surfaces in ordinary space in the late nineteenth century, provide an excellent instance of such a consistency. Thus, the investigations of the interplay between the discrete and the continuous integrable systems acquire a certain significance, and in our view, the role of the second example of a generating PDE, becomes key.

\section{The field equations and their linear representation}

We first note that the Euler-Lagrange equations associated with the Lagrangian $L$, admit of a linear representation. This means that the field equations are a compatibility condition for the existence of the solution of an auxiliary, linear, overdetermined system of equations of the form

$$
\psi_{, u}=A(u, v ; \lambda) \psi, \quad \psi_{, v}=B(u, v ; \lambda) \psi .
$$

The complex $r$-vector $\psi$ and the $r \times r$-matrices $A, B$ are functions of $u, v$ and a complex parameter $\lambda$, called the spectral parameter. The compatibility condition of the overdetermined linear system (5) is

$$
A_{, v}-B_{, u}+[A, B]=0,
$$

which should hold identically for every value of $\lambda$, where $[$,$] denotes usual matrix commutator.$ Let us now consider the case where $A$ and $B$ are rank 1 matrices and their sole dependence on the spectral parameter $\lambda$ is given by the following rational expressions:

$$
A_{i j}=\frac{a_{i} d_{j}}{u-\lambda}, \quad B_{i j}=\frac{b_{i} c_{j}}{v-\lambda},
$$

$i, j=0,1 \ldots r-1$. Then, equation (6) implies the existence of the parameter functions $n(u), m(v)$, introduced by

$$
n(u)=a^{i} d_{i}, \quad m(v)=b^{i} c_{i},
$$

$i=0,1, \ldots r-1$. Indices will be raised and lowered using the Kronecker delta symbol $\delta_{i j}$, and summation over repeated upper and lower indices is understood. Without loosing generality we may fix $a_{0}=b_{0}=1$. Consequently, equation (6) leads to the following system of PDEs:

$$
\begin{aligned}
(u-v) a_{i, v}-a^{j} c_{j}\left(a_{i}-b_{i}\right) & =0, \\
(u-v) b_{i, u}-b^{j} c_{j}\left(a_{i}-b_{i}\right) & =0, \\
(u-v) d_{i, v}+a^{j} c_{j} d_{i}-b^{j} d_{j} c_{i} & =0, \\
(u-v) c_{i, u}+a^{j} c_{j} d_{i}-b^{j} d_{j} c_{i} & =0,
\end{aligned}
$$

$i, j=0,1, \ldots r-1$. An obvious consequence of the latter system of PDEs is the relation $c_{i, u}=d_{i, v}$, which implies the existence of the functions $f_{i}$, such that

$$
c_{i}=f_{i, v} \quad d_{i}=f_{i, u}
$$

$i=0,1 \ldots r-1$. In terms of the new variables $f_{i}$ and using equation (8), the system of PDEs (9) -(12) reads

$$
\begin{aligned}
(u-v) a_{i, v}-\left(m+\left(a_{j}-b_{j}\right) f_{, v}^{j}\right)\left(a_{i}-b_{i}\right) & =0 \\
(u-v) b_{i, u}-\left(n-\left(a_{j}-b_{j}\right) f_{, u}^{j}\right)\left(a_{i}-b_{i}\right) & =0 \\
(u-v) f_{i, u v}+m f_{i, u}-n f_{i, v}+C_{i j}\left(a^{j}-b^{j}\right) & =0
\end{aligned}
$$


where $i, j=1,2, \ldots r-1$, and

$$
C_{i j}=f_{i, u} f_{j, v}+f_{i, v} f_{j, u} .
$$

We note that in general, the $(r-1) \times(r-1)$-matrix $C$ has rank 2 . In the following we consider the case $r=3$. In this case, by eliminating the variables $a_{i}, b_{i}$ from the system (14)-(16), we arrive at a coupled system of fourth order PDEs for the variables $f_{i}, i=1,2$. To this end, one solves the linear system (16) for the differences $a_{i}-b_{i} \equiv z_{i}$, in terms of the partial derivatives of the unknown functions $f_{i}$. Substituting the result into the remaining equations (14), (15) and using the compatibility condition $b_{i, u v}=b_{i, v u}$ (or equivalently $a_{i, u v}=a_{i, v u}$ ) we get

$$
D_{u} D_{v} z_{i}=D_{u}\left(\frac{z_{i}\left(z^{j} f_{j, v}+m\right)}{u-v}\right)+D_{v}\left(\frac{z_{i}\left(z^{j} f_{j, u}-n\right)}{u-v}\right)
$$

$i, j=1,2$, where $D$ denotes total differentiation with respect to the indicated arguments in the subscripts. The fourth order system of PDEs (18), is precisely the equations obtained by varying the fields $\left(f_{1}, f_{2}\right)$ in the Lagrangian $L$.

\section{Infinite symmetries and the Boussinesq hierarchy}

We next illustrate the infinite dimensional flows which are compatible with the linear representation (5), and consequently with the field equations. This amounts to finding and representing the infinite symmetries of the field equations. To this end we follow the construction given in [16], cf [17] in connection with techniques such as the vertex operator. Having found the infinite symmetries of the field equations, the representation is accomplished in three steps. First, by introducing an infinite set of indeterminates $x^{i}=\left(x^{1}, x^{2}, \ldots\right)$, which are the parameters of the group associated with the symmetry algebra. Next, by imposing certain linear differential relations for the potentials $\psi_{i}$ with respect to $x^{i}$, in such a way that each of these relations is compatible with the linear pair (5). Finally, by treating the indeterminates $x^{i}$ as additional independent variables, which leads naturally our considerations to the commuting compatible flows, i.e. an infinite compatible set of PDEs for $H\left(u, v, x^{i}\right)$.

In order to find the symmetries of the field equations (14)-(16), it is necessary to introduce the matrix-valued function $H$, as follows

$$
H_{i j, u}=a_{i} f_{j, u}, \quad H_{i j, v}=b_{i} f_{j, v},
$$

$i, j=0,1,2$. The existence of $H$ is guaranteed by the field equations (14)-(16), which now acquire the matrix form

$$
(v-u) H_{, u v}+\left[H_{, u}, H_{, v}\right]=0 .
$$

The symmetry algebra of equation (20) is generated by all characteristic vector fields

$$
X_{Q}=Q \partial_{H}+\left(D_{u} Q\right) \partial_{H_{, u}}+\left(D_{v} Q\right) \partial_{H_{, v}}+\left(D_{u} D_{v} Q\right) \partial_{H_{, u v}},
$$

for which the matrix-valued function $Q$ satisfies the linearized equation (20), namely

$$
(v-u) D_{u} D_{v} Q+\left[D_{u} Q, H_{, v}\right]+\left[H_{, u}, D_{v} Q\right]=0 .
$$

Equation (22) should hold for all solutions of equation (20), and as such the field equations and their differential consequences are taken into account. In general, the symmetry characteristic $Q$ 
depends on the coordinates $(u, v)$, on $H$ and its partial derivatives up to an unspecified order, and on potentials of $H$. If $Q$ is linear in the first partial derivatives of $H$, then equation (22) delivers the local, or Lie-point, symmetries of equations (20). These symmetries are given by the characteristic

$$
Q_{0}=\varepsilon_{1}([J, H]+K)+\varepsilon_{2}\left(H_{, u}+H_{, v}\right)+\varepsilon_{3}\left(u H_{, u}+v H_{, v}\right),
$$

where $J$ and $K$ are complex constant matrices and $\varepsilon_{i}$ complex constants. It should be noticed that if $n$ and $m$ are parameter functions of $u$ and $v$ respectively, then only the symmetry with $\varepsilon_{1} \neq 0$ and $\varepsilon_{2}=\varepsilon_{3}=0$, is compatible with the symmetries of the field equations (14)-(16), since on the contrary the characteristic corresponds to the affine base transformations (4). The following observation allows one to find in principle, an infinite number of symmetries of equations (20), starting from the characteristic $Q_{0}$ with $\varepsilon_{1}=1, \varepsilon_{2}=\varepsilon_{3}=0$.

Equation (22), can be written in the form

$$
D_{v}\left(u D_{u} Q+\left[Q, H_{, u}\right]\right)=D_{u}\left(v D_{v} Q+\left[Q, H_{, v}\right]\right)
$$

which implies the existence of the matrix-valued potential $\widetilde{Q}$ introduced by

$$
D_{u} \widetilde{Q}=u D_{u} Q+\left[Q, H_{, u}\right], \quad D_{v} \widetilde{Q}=v D_{v} Q+\left[Q, H_{, v}\right] .
$$

By direct calculations and using the Jacobi identity, one finds that the potential $\widetilde{Q}$ is also a symmetry of equations (20), whenever $Q$ and $H$ satisfy (22) and (20), respectively. In other words, equations (25) define a recursion operator $\mathcal{R}$, i.e. an operator with the property that whenever $Q$ is a characteristic of a symmetry of equations (20), so is $\widetilde{Q}=\mathcal{R} Q$. It follows from equations (25), that we may formally write $\mathcal{R}$ as the operator

$$
\mathcal{R}=\mathrm{D}^{-1} \cdot\left(\sigma \mathrm{D}-\varrho * \mathrm{D}+\operatorname{ad}_{\mathrm{d} H}\right) .
$$

Here, $\mathrm{D}$ and d denote total and ordinary differentials respectively, $*$ is the two dimensional Hodge duality operator which acts on the basis of one forms by

$$
* \mathrm{~d} u=\mathrm{d} u, \quad * \mathrm{~d} v=-\mathrm{d} v
$$

$\operatorname{ad}_{A}(B)=[B, A]$, and the functions $\varrho$ and $\sigma$ are given by

$$
\sigma=\frac{1}{2}(v+u), \quad \varrho=\frac{1}{2}(v-u) .
$$

From the above discussion it is clear that starting with specific matrices $J$ and $K$ and using the recursion relations

$$
Q_{p+1}=\mathcal{R} Q_{p}, \quad Q_{0}=[J, H]+K, \quad p \in \mathbb{N},
$$

we may generate an infinite number of symmetries of equations (20). Let $x^{p}=\left(x^{0}, x^{1}, \ldots\right), p \in \mathbb{N}$, denote the parameters of the infinitesimal symmetry transformations which are generated by the vector fields $X_{Q_{p}}$, i.e.

$$
\partial_{x^{p}} H=Q_{p}, \quad p \in \mathbb{N} .
$$

Since for every fixed $p$, the characteristic $Q_{p}$ defines a symmetry of equation (20), the pair of PDEs which is formed by taking each of equations (30) together with equation (20), is a compatible system of PDEs for $H\left(u, v, x^{p}\right)$ ( $p$ fixed). By virtue of this fact, every such pair of PDEs, admits 
also a linear representation. The latter is given by the linear equations (5) for $\psi$, and which for convenience we rewrite below

$$
(u-\lambda) \psi_{, u}=H_{, u} \psi, \quad(v-\lambda) \psi_{, v}=H_{, v} \psi,
$$

together with each of the following linear equations

$$
\begin{aligned}
\psi_{, x^{0}} & =J \psi, \\
\psi_{, x^{p+1}} & =-\lambda \psi_{, x^{p}}-Q_{p} \psi, \quad p \in \mathbb{N} .
\end{aligned}
$$

By induction, we may easily prove that the compatibility conditions $\partial_{x^{p}} \partial_{u} \psi=\partial_{u} \partial_{x^{p}} \psi$ and $\partial_{x^{p}} \partial_{v} \psi=$ $\partial_{v} \partial_{x^{p}} \psi$ are satisfied for every $p \in \mathbb{N}$, whenever $H$ satisfies equation (20) and $Q_{p}$ are the symmetry characteristics of the previous construction.

Similarly, starting with some other matrices $\widehat{J}$ and $\widehat{K}$, one obtains a different infinite hierarchy of symmeries of equation (20). Let $y^{q}=\left(y^{0}, y^{1}, \ldots\right), q \in \mathbb{N}$ denote the parameters of the corresponding symmetry transformations generated by the vector fields $X_{\widehat{Q}_{q}}$. In analogy with the preceding construction, the linear equations which can be imposed in addition to the linear equations (31) are

$$
\begin{aligned}
\psi_{, y^{0}} & =\widehat{J} \psi, \\
\psi_{, y^{q+1}} & =-\lambda \psi, y^{q}-\widehat{Q}_{q} \psi, \quad q \in \mathbb{N},
\end{aligned}
$$

We now assume that the potentials $H$ and $\psi$ depend in addition to $(u, v)$, on $x^{p}$ and also on $y^{q}$, $\forall p, q \in \mathbb{N}$. Since $x^{p}$ and $y^{q}$ will be treated as additional independent variables, it is necessary that

$$
\partial_{x^{p}} \partial_{y^{q}} F-\partial_{y^{q}} \partial_{x^{p}} F=0, \quad \forall p, q \in \mathbb{N} .
$$

holds, for all functions $F\left(u, v, x^{p}, y^{q}\right)$. The aim now is to generate the compatible set of PDEs for $H$, in terms of the higher time variables $x^{p}, y^{q}$. This infinite set is obtained by taking the compatibility conditions $\partial_{x^{p}} \partial_{y^{q}} \psi=\partial_{y^{q}} \partial_{x^{p}} \psi$, on the linear representations (32), (33) and (34), (35). In order to give an explicit description and for purposes of calculation we proceed as follows.

The leading equation (36) is valid for every solution $H$ of equation (20), whenever the starting matrices satisfy

$$
[J, \widehat{J}]=0, \quad \text { and } \quad[J, \widehat{K}]=[\widehat{J}, K] .
$$

By virtue of relations (37), we fix the values of the starting matrices as follows:

$$
\begin{array}{rlr}
\left(J_{i j}\right)=\left(\begin{array}{lll}
0 & 0 & 0 \\
0 & 0 & 0 \\
1 & 0 & 0
\end{array}\right), & \left(K_{i j}\right)=\left(\begin{array}{rrr}
0 & -1 & 0 \\
0 & 0 & -1 \\
0 & 0 & 0
\end{array}\right), \\
\left(\widehat{J}_{i j}\right)=\left(\begin{array}{lll}
0 & 0 & 0 \\
1 & 0 & 0 \\
0 & 1 & 0
\end{array}\right), & \left(\widehat{K}_{i j}\right)=\left(\begin{array}{rrr}
0 & 0 & -1 \\
0 & 0 & 0 \\
0 & 0 & 0
\end{array}\right),
\end{array}
$$

$i, j=0,1,2$. Next, we factor out the linear set of PDEs for $H$, which is obtained from the compatibility conditions $\partial_{x^{p}} \partial_{y^{q}} \psi=\partial_{y^{q}} \partial_{x^{p}} \psi$ with $p=0, q \geq 1,(q=0, p \geq 1)$. Finally, we combine the remaining linear representations (33) and (35) into one, by introducing (i) auxiliary higher time variables

$$
w^{i}=\left(w^{1}=x^{1}, w^{2}=y^{1}, w^{4}, w^{5}, w^{7} \ldots\right), \quad i \in \mathbb{Z} \backslash 3 \mathbb{Z}, \quad i \geq 1,
$$


and (ii) the matrix

$$
\Lambda=-\lambda J-K .
$$

From this starting point, and noticing that

$$
\Lambda^{2}=-\lambda \widehat{J}-\widehat{K}
$$

the linear infinite set of differential relations for $\psi$, which can be simultaneously imposed on the linear representation (31), is given by

$$
\begin{aligned}
\psi_{, w^{1}} & =\left(\Lambda+\left[\Lambda^{\prime}, H\right]\right) \psi, \\
\psi_{, w^{2}} & =\left(\Lambda^{2}+\left[\left(\Lambda^{2}\right)^{\prime}, H\right]\right) \psi, \\
\psi_{, w^{i+3}} & =-\lambda \psi_{, w^{i}}-H_{, w^{i}} \psi, \quad i \in \mathbb{Z} \backslash 3 \mathbb{Z}, \quad i \geq 1,
\end{aligned}
$$

where prime in equations (43), (44) denotes differentiation with respect to $\lambda$. The above differential relations generate an infinite set of PDEs for $H$, with the Boussinesq equation being the first member. Specifically, the compatibility condition $\partial_{w^{2}} \partial_{w^{1}} \psi=\partial_{w^{1}} \partial_{w^{2}} \psi$ on equations (43), (44), yields a set of six, first order PDEs for the components of $H$. After a simple differentiation and elimination process applied to the latter system, and using (19) to identify the main field variables $\left(f_{1}, f_{2}\right)$, we arrive at the following system of PDEs:

$$
\begin{aligned}
& f_{1, x y}=2 f_{2, y y}+\left(f_{2} f_{2, x}\right)_{, y}-6 f_{2, x} f_{2, x x}-\frac{1}{2} f_{2, x x y}+\frac{1}{4} f_{2, x x x x}, \\
& f_{1, x x}=\left(f_{2} f_{2, x}\right)_{, x}+\frac{1}{2}\left(f_{2, x y}-f_{2, x x x}\right)
\end{aligned}
$$

where $x=w^{1}, y=w^{2}$. Finally, taking the compatibility condition $f_{1, x y x}=f_{1, x x y}$ on equations (46), (47) we obtain

$$
3 \mathcal{B}_{, y y}+\mathcal{B}_{, x x x x}+6\left(\mathcal{B}^{2}\right)_{, x x}=0,
$$

where $\mathcal{B}=-f_{2, x}$. Equation (48) is known as the Boussinesq equation. Similarly, higher members of the Boussinesq hierarchy can be derived from the system (43)-(45), in a systematic way.

\section{A Bäcklund auto-transformation}

The discussion in the present section relates to matters that had their origin in the investigations by Bäcklund near the end of the nineteenth century, concerning the transformation properties of simultaneous partial differential equations and the transformation theory of surfaces in ordinary space. There are many methods developed over the past century to obtain the Bäcklund transformations for certain classes of partial differential equations, see [18, 19, 20, 21] for recent advances on these topics. However, for the construction of a Bäcklund auto-transformation for the relevant PDEs of our discussion, i.e. a transformation within the space of solutions of PDEs (14)-(16), we find the connection with the lattice Boussinesq equation, as a consequence of its three dimensional consistency, as the most powerfull.

An upshot of recent investigation of the equations (18), and their compatible discrete counterparts, has been the insight that both systems arise as parameter families of mutually consistent equations. In turn, these equations form multi-variable and multi-parameter systems, in which parameters and variables play a dual (even interchangeable) role, and the whole infinite system is characterized by an intrinsic consistency. In the light of the close relation of equation (18) 
with the Ernst equations, as will be explained in the next section, and since solution generating techniques such as Bäcklund transformations, have proven to be invaluable methods in finding physically significant exact solutions of the Ernst equations [22, 23, 24, we now explain how a Bäcklund auto-transformation for equations (18) can be constructed straightforward, by exploiting the consistency approach.

The construction is based on the compatible discrete system as this was derived in [25. In order to make contact with the latter system, it is necessary to retract for the moment the dependence of the parameter functions $n, m$ on $u, v$, respectively. ${ }^{1}$ Let us now consider an elementary plaquette of a two-dimensional lattice, as shown in figure 1. The dependent variables (fields) are assigned on the vertices at sites $(n, m)$, and $(u, v)$ which now play the role of the lattice parameters, are assigned on the edges. The updates of a lattice variable $F$ along a shift in the $n$ and $m$ direction of the lattice will be denoted by $F_{[1]}, F_{[2]}$ respectively, i.e.

$$
F_{[1]}=F(n+1, m), \quad F_{[2]}=F(n, m+1), \quad F_{[12]}=F(n+1, m+1) .
$$

The partial difference equations which are compatible with the partial differential equations (14)-

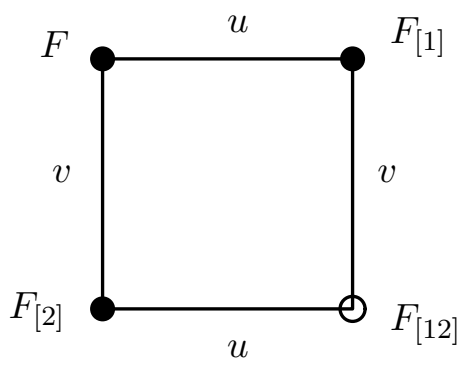

Figure 1: An elementary quadrilateral for the discrete Boussinesq system.

(16), in the sense that these two systems share a common set of solutions, is given by the following parameter family of algebraic equations:

$$
\begin{aligned}
W_{[1]} & =U U_{[1]}-V, \\
W_{[2]} & =U U_{[2]}-V, \\
W & =U U_{[12]}-V_{[12]}+\frac{u-v}{U_{[2]}-U_{[1]}} .
\end{aligned}
$$

The system of equations (501)-(52), relates the values of the fields assigned on the four vertices of the quadrilateral, where now $F=(U, V, W)$, and is called the discrete Boussinesq system. For the Cauchy problem on a staircase, we should impose on the black points of the elementary plaquette of figure 1 , the initial values $(U, V, W),\left(U_{[1]}, V_{[1]}\right),\left(U_{[2]}, V_{[2]}\right)$ only, since from these data and the discrete equations (50])-(52), the values $\left(W_{[1]}, W_{[2]}\right)$ and $\left(U_{[12]}, V_{[12]}, W_{[12]}\right)$ are determined uniquely. In particular, using equations (50), (51) we find that

$$
U_{[12]}=\frac{V_{[1]}-V_{[2]}}{U_{[1]}-U_{[2]}},
$$

\footnotetext{
${ }^{1}$ Relative to the continuous field equations, this restriction is not essential since the Bäcklund transformation that we derive below, is also valid with $n, m$ parameter functions.
} 
and subsequently the values $W_{[12]}$ and $V_{[12]}$ can be found uniquely, from equation (50) (or (51)) and equation (52), respectively. The fields which are assigned on the vertices with black points, are identified with the fields of the continuous PDEs, as follows:

$$
\begin{aligned}
W & =f_{1}, \quad U=-f_{2}, \quad V=-H_{12}, \\
U_{[1]} & =a_{1}, \quad U_{[2]}=b_{1} \quad V_{[1]}=a_{2}, \quad V_{[2]}=b_{2} .
\end{aligned}
$$

In [25] it is shown that the discrete Boussinesq system represents a consistent parameter family of two-dimensional lattice equations, in the sense that equations (150)-(152) can be imposed on all faces of a three-dimensional lattice in a consistent way. The third direction of the generated lattice has a dual role. On the discrete level, is taken as auxiliary (spectral) and the system is linearized in the corresponding variables $U_{[3]}, V_{[3]}$ and their shifts. On the other hand, the discrete equations which relate the fields and their shifts in the third direction, define a map in the solution space of the continuous field equations, by virtue of the identifications (54)-(55).

Specifically, extending equations (50)-(52) into a third direction associated with a new lattice parameter (identified here with the spectral parameter $\lambda$ ) we get

$$
\begin{array}{rlr}
W_{[3]}=U U_{[3]}-V, & \\
U_{[13]}=\frac{V_{[1]}-V_{[3]}}{U_{[1]}-U_{[3]}}, & W=U U_{[13]}-V_{[13]}+\frac{u-\lambda}{U_{[3]}-U_{[1]}}, \\
U_{[23]}=\frac{V_{[2]}-V_{[3]}}{U_{[2]}-U_{[3]}}, & W=U U_{[23]}-V_{[23]}+\frac{v-\lambda}{U_{[3]}-U_{[2]}} .
\end{array}
$$

The auxiliary variables $U_{[3]}, V_{[3]}$ are identified as projective variables and their relation with the spectral variables $\psi_{0}, \psi_{1}, \psi_{2}$ is given by

$$
U_{[3]}=\frac{\psi_{1}}{\psi_{0}}, \quad V_{[3]}=\frac{\psi_{2}}{\psi_{0}} .
$$

By straightforward calculations one easily verifies that the linear system (5) for the potentials $\left(\psi_{0}, \psi_{1}, \psi_{2}\right)$, implies the Riccati system for the potentials $\left(q_{1}, q_{2}\right) \equiv\left(U_{[3]}, V_{[3]}\right)$,

$$
\begin{aligned}
(u-\lambda) q_{i, u} & =\left(a_{i}-q_{i}\right)\left(n-\left(a_{j}-q_{j}\right) f_{, u}^{j}\right), \\
(v-\lambda) q_{i, v} & =\left(b_{i}-q_{i}\right)\left(m-\left(b_{j}-q_{j}\right) f_{, v}^{j}\right),
\end{aligned}
$$

$i=1,2$. In virtue of the identifications (54)-(55), we now interpret the shift of the fields in the third direction, as a map $\varphi$ in the solution space of equations (14)-(16). Explicitly, using equations (57) -(58) and the identifications (54)-(55), the map $\varphi$ is defined by

$$
\begin{array}{llll}
\varphi\left(f_{1}\right) & =H_{12}-f_{2} q_{1}, & \varphi\left(f_{2}\right) & =-q_{1}, \\
\varphi\left(a_{1}\right) & =\frac{a_{2}-q_{2}}{a_{1}-q_{1}}, & \varphi\left(a_{2}\right) & =-f_{1}-f_{2} \varphi\left(a_{1}\right)+\frac{u-\lambda}{q_{1}-a_{1}}, \\
\varphi\left(b_{1}\right) & =\frac{b_{2}-q_{2}}{b_{1}-q_{1}}, & \varphi\left(b_{2}\right) & =-f_{1}-f_{2} \varphi\left(b_{1}\right)+\frac{v-\lambda}{q_{1}-b_{1}} .
\end{array}
$$

Restricting now our considerations on the continuum level, the above equations define a Bäcklund auto-transformation, i.e. a transformation that generates new solutions of the field equations from 
other known ones. Below we summarize the resulting solution generating procedure, which can be verified by lenghy but straightforward calculations.

Let $\left(f_{1}, f_{2}\right)$ be a known solution of equations (18). From this solution and using equations (14)-(16), one determines by quadrature the corresponding vector components $\left(a_{1}, a_{2}\right)$ and $\left(b_{1}, b_{2}\right)$, up to constants of integration. Successively, the auxiliary complex potentials $H_{12}$ and $\left(q_{1}, q_{2}\right)$ can be found by quadrature from equation (19), and by integrating the Riccati system (60)-(61), respectively. Then, a new solution $\left(\widetilde{a}_{1}, \widetilde{a}_{2}\right),\left(\widetilde{b}_{1}, \widetilde{b}_{2}\right)$ and $\left(\widetilde{f}_{1}, \widetilde{f}_{2}\right)$ of the system (14) $-(\underline{16})$, and a fortiori for the equations (18), is given by the algebraic relations (62)-(64), where $\widetilde{f}_{i}=\varphi\left(f_{i}\right), \widetilde{a}_{i}=\varphi\left(a_{i}\right)$, $\widetilde{b}_{i}=\varphi\left(b_{i}\right), i=1,2$.

\section{Reduction to the Ernst equations}

We next show that the main second order subsystem of PDEs which is incorporated into the fourth order system of equations (18), is the hyperbolic Ernst equations for an Einstein-MaxwellWeyl field. In order to make this connection more transparent, we introduce auxiliary complex potentials $\left(h_{1}, h_{2}\right)$ by the relations

$$
h_{1}=\frac{a_{2}-b_{2}}{a_{1}-b_{1}}, \quad h_{2}=\frac{b_{1} a_{2}-a_{1} b_{2}}{a_{1}-b_{1}} .
$$

and which correspond to a change of the variables $\left(a_{2}, b_{2}\right)$, of the form

$$
a_{2}=h_{1} a_{1}+h_{2}, \quad b_{2}=h_{1} b_{1}+h_{2} .
$$

It follows from the system of equations (14), (15) that

$$
a_{2, v}=h_{1} a_{1, v}, \quad b_{2, u}=h_{1} b_{1, u},
$$

which in virtue of equations ([66), yield

$$
h_{2, v}=-h_{1, v} a_{1}, \quad h_{2, u}=-h_{1, u} b_{1} .
$$

From this starting point, and eliminating the variable $f_{1}$ from the field equations (14)-(16), we may recast the latter system as a coulped set of four PDEs of second order in terms of the variables $\left(a_{1}, b_{1}, h_{1}, f_{2}\right)$. This system of PDEs is given by

$$
\begin{aligned}
a_{1, u v} & =2 \frac{a_{1, u} a_{1, v}}{a_{1}-b_{1}}-\frac{m}{u-v} a_{1, u}-\frac{n+1}{u-v} a_{1, v}+\frac{h_{1, u} f_{2, v}}{u-v}\left(a_{1}-b_{1}\right)^{2}, \\
b_{1, u v} & =2 \frac{b_{1, u} b_{1, v}}{b_{1}-a_{1}}+\frac{m+1}{u-v} b_{1, u}+\frac{n}{u-v} b_{1, v}-\frac{h_{1, v} f_{2, u}}{u-v}\left(a_{1}-b_{1}\right)^{2}, \\
f_{2, u v} & =\frac{1}{a_{1}-b_{1}}\left(b_{1, u} f_{2, v}-a_{1, v} f_{2, u}\right), \\
h_{1, u v} & =\frac{1}{a_{1}-b_{1}}\left(b_{1, v} h_{1, u}-a_{1, u} h_{1, v}\right),
\end{aligned}
$$

where the first three PDEs follow from the system (14)-(16), and equation (72) follows from the compatibility condition $h_{2, u v}=h_{2, v u}$ on equations (68).

Let now $u, v$ be real valued, and $\star$ denote complex conjugation. It follows from the system of PDEs (69)-(72), that the latter admits a compatible reduction by applying the reality conditions

$$
a_{1}=-b_{1}^{\star}, \quad h_{1}=f_{2}^{\star},
$$


whenever the parameter functions $n, m$ satisfy

$$
n+n^{\star}+1=0, \quad m+m^{\star}+1=0 .
$$

The resulting set of equations (69) and (71), which is formed by applying the above conditions, are the Ernst equations for an Einstein-Maxwell-Weyl field. A more standard form of the latter equations is given by a dual system, which is obtained here by rewriting the system (14)-(16) in terms of the above reality conditions. These conditions imply the following constraints on the variables $\left(a_{i}, b_{i}\right)$ :

$$
a_{1}=b_{1}+\frac{v-u}{f_{1}+f_{1}^{\star}+f_{2} f_{2}^{\star}}, \quad a_{2}=b_{2}+\frac{(v-u) f_{2}^{\star}}{f_{1}+f_{1}^{\star}+f_{2} f_{2}^{\star}} .
$$

By virtue of (75), one readily notices that the system of equations (14)-(16), decouples for the variables $\left(f_{1}, f_{2}\right)$, leading to a second order system. In order to make contact with the notation employed in general relativity, we relabel the main complex variables as $\left(f_{1}, f_{2}\right) \equiv(E, \Phi)$. Using equation (75) and adopting a coordinate free setting, the relevant second order system of PDEs is given by equations (16), and reads

$$
\begin{aligned}
\left(E+E^{\star}+\Phi \Phi^{\star}\right)(\mathrm{d}(\varrho * \mathrm{~d} E)-\mathrm{i} \boldsymbol{\beta} \wedge \mathrm{d} E) & =2 \varrho\left(\mathrm{d} E+\Phi^{\star} \mathrm{d} \Phi\right) \wedge * \mathrm{~d} E, \\
\left(E+E^{\star}+\Phi \Phi^{\star}\right)(\mathrm{d}(\varrho * \mathrm{~d} \Phi)-\mathrm{i} \boldsymbol{\beta} \wedge \mathrm{d} \Phi) & =2 \varrho\left(\mathrm{d} E+\Phi^{\star} \mathrm{d} \Phi\right) \wedge * \mathrm{~d} \Phi .
\end{aligned}
$$

Here, $\mathrm{i}=\sqrt{-1}$ and the one-form $\boldsymbol{\beta}$ is given by

$$
\boldsymbol{\beta}=\nu(u) \mathrm{d} u+\mu(v) \mathrm{d} v
$$

where the real parameter functions the $\nu, \mu$, are introduced by recasting the parameter constraints (74) in the form

$$
n=-\frac{1}{2}+\mathrm{i} \nu(u), \quad m=-\frac{1}{2}+\mathrm{i} \mu(v) .
$$

In the context of general relativity, equations (76), (77) are known as the Ernst equations for an Einstein-Maxwell-Weyl field [26]. It should be clear from the preceding discussion that, all solutions of the Ernst equations are embedded into the solution space of the Euler-Lagrange equations (18), while the converse does not hold in general. Therefore, equations (18), or equivalently the auxiliary system (14)-(15), represent a generalization of the Ernst equations (76), (77).

At this point, it is worth noticing that after applying the conditions (73), (79) and their consequences to the linear representation (5), the latter reduces to the one introduced in [26, 22, 28].

\section{Discussion}

Apart from the Ernst equations, which possess a prominent position among the subsystems included into the solution space of equations (18), there are also various interesting subsystems which are tied in with the latter set of equations. Thereof, we point out that equations (18), incorporate also the PDE which encodes the KdV hierarchy. This is accomplished by considering the case, $f^{1}=f^{2} \equiv f$, which leads to a scalar fourth order PDE for the variable $f$. The latter equation is the Euler-Lagrange equations for the Lagrangian density

$$
\mathcal{L}=(u-v) \frac{\left(f_{, u v}\right)^{2}}{f_{, u} f_{, v}}+\frac{1}{u-v}\left(m^{2} \frac{f_{, u}}{f_{, v}}+n^{2} \frac{f_{, v}}{f_{, u}}\right) .
$$


In [12, 13] it was shown that in the case where $n$ and $m$ are constants, the corresponding PDE for $f$, admits similarity solutions built from solutions of the full Painlevé VI (PVI) equation, i.e. with all parameters free. Alternatively, full PVI can be shown to arise naturally as symmetry reduction from the anti-self-dual Yang-Mills equations [29], or from the Ernst equation for an Einstein-Weyl field [30. Taking into account the richness of equations (18), it is even more interesting to consider their similarity reductions. Such a reduction leads to a six-parameter, second order, coupled system of ordinary differential equations [25], which we expect to be associated with the hierarchies of the PVI equation 31].

\section{Acknowledgments}

The initial stage of the work of A.T. was supported by the University of Patras research project C. Carathéodory, 2001/2785 and successively and mainly by the European postdoctoral fellowship Marie Curie, contract No HPMF-CT-2002-01639. The authors thank the referee for helpful suggestions.

\section{References}

[1] F.J. Ernst, New formulation of the axially symmetric gravitational field problem. Phys. Rev. 167 (1968), 1175-1178.

[2] F.J. Ernst, New formulation of the axially symmetric gravitational field problem. II. Phys. Rev. 168 (1968), 1415-1417.

[3] J.B. Griffiths, Colliding plane waves in general relativity. Oxford University Press, Oxford, 1991.

[4] A.S. Fokas, L-Y Sung and D. Tsoubelis, The inverse spectral method for colliding gravitational waves. Math. Phys., Anal. and Geom. 1 (1999), 313-330.

[5] I. Hauser and F.J. Ernst, Proof of a generalized Geroch conjecture for the hyperbolic Ernst equation. Gen. Relativ. Gravit. 33 (2001), 195-293.

[6] G.A. Alekseev, A new integral equation form of integrable reductions of the Einstein equations. Theor. Math. Phys. 129 (2001), 1466-1483.

[7] G.A. Alekseev and J.B. Griffiths, Solving the characteristic initial-value problem for colliding plane gravitational and electromagnetic waves. Phys. Rev. Let. 87 (2001) 221101-221104.

[8] R. Geroch, A method for generating solutions of Einstein's equations. J. Math. Phys. 12 (1971), 918-924.

[9] R. Geroch, A method for generating new solutions of Einstein's equation. II. J. Math. Phys. 13 (1972), 394-404.

[10] W.K. Schief, A unified approach to infinitesimal Loewner and Geroch transformations and the Ernst and Einstein-Maxwell equations. J. Phys. A 34 (2001), 8659-8671.

[11] V.G. Kac, Infinite dimensional Lie algebras. Cambridge University Press, third edition, Cambridge, 1990. 
[12] F. Nijhoff, A. Hone and N. Joshi, On a Schwarzian PDE associated with the KdV hierarchy. Phys. Lett. A 267 (2000), 147-156.

[13] A. Tongas D. Tsoubelis and P. Xenitidis, A family of integrable nonlinear equations of hyperbolic type. J. Math. Phys. 42 (2001), 5762-5784.

[14] P.G. Olver, Equivalence, Invariants, and Symmetry. Cambridge University Press, Cambridge, 1995.

[15] V.E. Adler, A.I. Bobenko and Yu. B. Suris, Classification of Integrable Equations on QuadGraphs. The Consistency Approach. Commun. Math. Phys. 233 (2003), 513-543.

[16] F.W. Nijhoff, Linear integral transformations and hierarchies of integrable nonlinear evolution equations. Physica 31D (1988), 339-388.

[17] F.W. Nijhoff, Integrable hierarchies, Lagrangians structures and non-commuting flows. in Topics in soliton theory and exactly solvable nonlinear equations, (Oberwolfach, 1986), World Sci. Publishing, Singapore, 150-181, 1987.

[18] R.L. Anderson and N.H. Ibragimov, Lie-Bäcklund transformations in Applications. Soc. Ind. Appl. Math., Philadelphia, 1979.

[19] C. Rogers and W.F. Shadwick, Bäcklund transformations and their applications. Academic Press, New York, 1982.

[20] V.B. Matveev and M.A. Salle, Darboux transformations and solitons. Springer-Verlag, Berlin, 1991.

[21] C. Rogers and W.K. Schief, Bäcklund and Darboux transformations, Geometry and modern applications in soliton theory. Cambridge University Press, Cambridge, 2002.

[22] B.K. Harrison, Bäcklund transformation for the Ernst Equation of general relativity. Phys. Rev. Let. 41 (1978), 1197-1200.

[23] G. Neugebauer, Bäcklund transformations of axially symmetric stationary gravitational fields. J. Phys. A. 12 (1979), L67-L70.

[24] D. Kramer and G. Neugebauer, Prolongation structure and linear eigenvalue equations for Einstein-Maxwell fields. J. Phys. A. 14 (1981), L333-L338.

[25] A. Tongas and F. Nijhoff, The Boussinesq integrable system: Compatible lattice and continuum structures. Glasgow Math. J. to appear (2005).

[26] G.A. Alekseev, Soliton configurations of interacting massless fields. Sov. Phys. Dokl. 28 (1983), 133-135.

[27] G.A. Alekseev, The method of the inverse scattering problem and singular integral equations for interacting massless fields. Sov. Phys. Dokl. 30 (1985), 565-568.

[28] G.A. Alekseev, Exact solutions in the general theory of relativity. Proc. Steklov Inst. Math. 3 (1988), 215-262. 
[29] L.J. Mason and N.M.J. Woodhouse, Integrability Self-Duality, and Twistor Theory. Oxford University Press, Oxford, 1996.

[30] W.K. Schief, The Painlevé III, V and VI transcendents as solutions of the Einstein-Weyl equations. Phys. Lett. A 267 (2000), 265-275.

[31] F.W. Nijhoff and A.J. Walker, The discrete and continuous Painlevé VI hierarchy and the Garnier systems. Glasgow Math. J. 43A (2001), 109-123. 